\title{
3 Research Square

\section{Treatment outcomes of drug-susceptible tuberculosis and its predictors among male prison inmates in Bauchi State, Nigeria, 2014-2018}

Peter Okpeh Amede ( $\square$ perosports05@gmail.com )

African Field Epidemiology Network https://orcid.org/0000-0002-9200-0379

Elizabeth Adedire

African Field Epidemiology Network

Aishat Usman

African Field Epidemiology Network

Celestine A. Ameh

African Field Epidemiology Network

\section{Faruk S. Umar}

Nigerian Prisons Service

Chukwuma David Umeokonkwo

Department of community medicine, Alex Ekwueme Federal University Teaching Hospital Abakiliki, Ebonyi State, Nigeria

Muhammad Shakir Balogun

African Field Epidemiology Network

\section{Research article}

Keywords: Tuberculosis, Treatment outcomes, Prison inmates, Predictors, Bauchi State, Nigeria

Posted Date: November 19th, 2019

DOI: https://doi.org/10.21203/rs.2.17443/v1

License: (c) (1) This work is licensed under a Creative Commons Attribution 4.0 International License. Read Full License 


\section{Abstract}

Background: Tuberculosis (TB) is a contagious disease and its transmissibility potential is increased in congregate settings like the prisons. TB incidence rates are five to fifty times higher among prison inmates than the general population which has a direct impact on the outcome of TB treatment. There is paucity of information on TB treatment outcomes and its predictors in Nigerian prisons. We therefore assessed TB treatment outcomes among prison inmates in Bauchi State, Nigeria. Method: We conducted a retrospective data analysis of inmates with TB in the five main prisons in Bauchi State. We extracted sociodemographic, clinical and treatment outcome characteristics from TB treatment register of inmates treated for TB between January 2014 and December 2018, using a standardized checklist. We estimated the TB treatment success rate (TSR) and explored the relationship between the TSR and sociodemographic and clinical characteristics. Related variables were modelled in multivariate logistic regression to identify predictors of TSR at $5 \%$ level of significance. Results: All 216 inmates were male with mean age of $37.6 \pm 11.4$ years. Seventy-six (35.2\%) were cured, 61 (28.2\%) completed treatment, 48 (22.2\%) were lost to follow-up, 17 (7.9\%) were transferred out without evaluation and $14(6.5 \%)$ died. Overall TSR was $72.9 \%$. Odds of successful treatment outcome were age; $20-29$ years (AOR=10.5; $95 \% \mathrm{Cl}$ : 3.2-35.1), 30-39 years (AOR=4.2; 95\% Cl: 1.3-13.1), pretreatment weight; $50-59 \mathrm{~kg}$ (AOR= 9.6; 95\% Cl: 1.465.6), $\geq 60 \mathrm{~kg}$ ( $A O R=18.6 ; 95 \% \mathrm{Cl}: 2.5-140.1$ ) and being HIV negative ( $\mathrm{AOR}=3.3 ; 95 \% \mathrm{Cl}: 1.4-7.8)$. Conclusion: The predictors of successful TB treatment outcome were being less than 40 years of age, having a pretreatment body weight of or greater than $50 \mathrm{~kg}$, imprisonment for less than 2 years, and being HIV negative. We recommended that to improve TB TSR among prison inmates; age, duration of imprisonment, weight and TB/HIV coinfection should be the major consideration during drugs adherence, psychological and nutritional counselling and a tracking system be developed by the prisons authority to follow-up inmates transferred-out to other health facilities to ensure they complete the treatment and outcomes evaluated. Key words: Tuberculosis, Treatment outcomes, Prison inmates, Predictors, Bauchi State, Nigeria

\section{Background}

Tuberculosis (TB) is a preventable and curable disease with effective drugs and vaccines, despite this it remain a major killer globally with over 4,500 dying from the infection daily. ${ }^{1,2}$ It was the $10^{\text {th }}$ leading cause of death; with 1.6 million deaths attributed to the disease and 10 million cases reported worldwide in 2017. ${ }^{3}$ Nigeria ranked $7^{\text {th }}$ in the world and $2^{\text {nd }}$ in Africa among the 30 countries with the highest burden of TB, in 2017. ${ }^{3}$ The high prevalence of TB is driven by HIV infection, lack of TB diagnostic laboratories, poverty and weak healthcare systems. ${ }^{4}$

Tuberculosis transmissibility potential is increased in congregate settings such as prisons due to a high prevalence of HIV infection among prison inmates, overcrowding, poor nutrition, poor hygiene, prolonged indoor confinement without adequate ventilation and limited access to healthcare. ${ }^{5-8}$ Overcrowding in Nigerian prisons has been on a steady rise; the total prison population in 2018 was 73,631 up from 
44,450 in 2000 and 57,313 in 2015 without a corresponding expansion of the capacity of the prisons. ${ }^{9}$ Globally, TB burden among prison inmates is $5-50$ times higher than among the general population and is estimated to be the leading cause of death among prison inmates. A case of active Pulmonary TB (PTB) can infect 10-15 persons over the course of a year, this might be higher among prison population due to overcrowding and prolonged close contacts. ${ }^{10-11}$ In sub-Saharan African prisons, TB remains one of the fastest growing infectious diseases. ${ }^{11-13}$

The goal of TB treatment is to cure those infected, prevent deaths from the disease and stop transmission of tubercle bacilli from infected individual to the host community. ${ }^{14}$ Tuberculosis treatment outcome is the best measure of the effectiveness of TB prevention and control programmes. ${ }^{15}$ Tuberculosis treatment outcome is classified as cured, treatment completed, treatment failed, lost to follow-up, transferred out and died. ${ }^{16,17}$ The treatment outcome is influenced by socio-demographic characteristics, socio-economic factors (such as poverty, housing), nutrition, HIV coinfection, Multi-Drugs Resistant TB (MDR-TB), and strategies for TB management including Directly Observed Treatment short course. $^{2,18,19}$

Tuberculosis treatment success is the sum of cured and treatment completed, and unsuccessful TB treatment outcome is the sum of treatment failure, lost to follow-up, transferred out and died. Treatment Success Rate (TSR) is the proportion of new and retreatment smear positive TB cases in a given year that successfully completed treatment with bacteriological evidence (cured) or without bacteriological evidence (treatment completed) of success among all who commenced the treatment. World Health Organization (WHO) set $\geq 90 \%$ as the global target for TSR to eliminate TB as a global public health concern and a cure rate of $\geq 85 \% .{ }^{17}$ The TB TSR globally was $83 \%$ in 2017 and the corresponding TSR for sub-Saharan Africa was $76 \%{ }^{17,18}$ In 2015, TB TSR in Nigeria was $84 \%$ below the WHO target, and ranked $84^{\text {th }}$ in the global rating and $23^{\text {rd }}$ in Africa. ${ }^{20}$

Prison inmates are more vulnerable to TB infection, and this has a direct impact on successful TB control in prisons and potential of disrupting the TB control programme in the non-incarcerated population. TB TSR among prison inmates in the European region in 2015 was 59.7\%, likewise a study in 2017 among 162 prison inmates with TB in Ethiopia, reported TSR of $63.62 \% .{ }^{21,23}$ No available data for TB TSR among prison population in Nigeria.

The prison population is a dynamic and unstable one and this might impact negatively on TB treatment outcomes. Poorly treated TB case in the prison could compound TB burden with-in the prison and increase the risk of MDR-TB outbreaks in the general population; however, there is limited information about TB treatment outcome among prison inmates in Nigeria. We therefore conducted this study to assess TB treatment outcomes and its predictors among prison inmates in Bauchi State, Nigeria.

\section{Methods}




\section{Study setting}

The study was conducted in all the five main prisons (Bauchi, Azare, Ningi, Misau and Jama'are) in Bauchi State. These prisons lock-up above its maximum capacity and the holding cells are usually overcrowded. The prisons have clinics with various cadre of healthcare workers (Doctor, nurses, community health officers, pharmacy assistant, dental assistant, community health extension workers, laboratory technologists and radiographer) that provides mainly curative services for inmates, staff and staff relations. These prisons have no diagnostic capacity for TB. On reception into the prisons, inmates are not offered screening for active tuberculosis. Those suspected to have TB were referred to health facility close to the prison for evaluation and on diagnosis, they are placed on Anti-TB drugs for the full course. Their treatment was continued in the prisons under the supervision of the prison health personnel.

\section{Study design and population}

We conducted a review of the treatment outcomes of all prison inmates treated for TB from January 2014 to December 2018 in all five prisons in Bauchi State. All prison inmates treated for TB with Anti-TB drugs from January 2014 to December 2018 were included. Inmates with missing variable(s) of interest in the TB treatment register were excluded (Figure 1).

\section{Data tool and collection}

The data source was the TB treatment register and patient health records in the five selected prisons. Data were extracted using a structured checklist. The checklist collected information on age, pretreatment weight, duration of imprisonment, TB class (Smear Positive Pulmonary Tuberculosis [SPPTB], Smear Negative Pulmonary Tuberculosis [SNPTB], Extra-Pulmonary Tuberculosis [EPTB]), treatment category (New, Retreatment, Unknown), HIV status, treatment outcomes (cured, treatment completed, failure, lost to follow-up, transferred out and died), from the TB treatment registers in the various study prisons by five trained healthcare workers (HCWs). The five HCWs (one from each prison) were trained for a day on how to collect the data and use the checklist. The principal researcher daily reviewed the filled format and strictly supervised the trained research assistants. Data were collected over a period of five weeks. Each prison was assigned a week; this enable the researcher to supervised the process of data abstraction.

\section{Measurement}

The dependent variable (treatment outcome) was dichotomized as successful (cured and treatment completed) and unsuccessful (treatment failure, lost to follow-up, transferred out and died) and the 
independent variables were socio-demographic characteristics (age, weight, duration of incarceration) and clinical characteristics (HIV status, TB class and treatment history). The following operational definitions were adopted for drug-susceptible TB. ${ }^{21}$

Cured: A PTB case with bacteriologically confirmed TB at the beginning of treatment who was smear or culture negative in the last month of treatment and on at least one previous occasion.

Treatment completed: A TB case who completed treatment without evidence of failure but without records to show that sputum smear or culture results in the last month of treatment and on at least one previous occasion were negative, either because tests were not done, or results were unavailable.

Treatment failure: A TB case whose sputum smear or culture is positive at month 5 or later during treatment.

Lost to follow-up: A TB case who did not start treatment or whose treatment was interrupted for two consecutive months or more.

Not evaluated: A TB case for whom no treatment outcome is assigned. This includes cases transferred out to another treatment unit as well as cases for whom the treatment outcome is unknown to the reporting unit.

Died: A TB case who dies for any reason during treatment.

New TB patient: A TB case who has not previously been treated for TB and is now diagnosed and has started the current treatment.

Relapse/Retreatment: A TB case who was previously treated for TB and was declared cured and now diagnosed and started the current treatment.

\section{Data analysis}

Extracted data were checked for its completeness, correctness and analyzed using Epi-info software version 7.2.2.6. Descriptive statistics was used to generate summary frequencies, percentages, and means. Bivariate analysis was performed to measure association between treatment outcome and independent variables and variables that were significant at bivariate analysis were included in multivariate logistic regression model to identify predictors of treatment outcomes at $95 \%$ confidence intervals (Cls).

\section{Ethical consideration}


Ethical approval was obtained from the Bauchi State Health Research Ethics Committee. Permission was sought and obtained from the Controller of Prisons, Bauchi State command, where the aim and objectives of the study were explained. The information obtained was made anonymous and de-identified prior to analysis to ensure confidentiality.

\section{Results}

Out of 228 TB patients treated for TB during the period, only 216 (95\%) had complete variable of interest and were therefore used for the analysis (Figure 1).

The mean age was $37.6 \pm 11.4$ years. The mean weight was $55.2 \pm 9.8 \mathrm{~kg}$ with $89(41.2 \%)$ having weight 50 $59 \mathrm{~kg}, 97$ (44.9\%) were incarcerated in Bauchi prison, the median (IQR) duration of imprisonment was 21 months (14-28 months) and 134 (62.0\%) were imprisoned for less than 2 years (Table 1). One hundred and eighty-eight (87.0\%) were newly diagnosed, 152 (70.3\%) were SPPTB and 46 (21.3\%) TB patients were co-infected with HIV (Table 2).

Seventy-six (35.2\%) were cured while 61 (28.2\%) had completed treatment, with no treatment failure recorded during the study period. Forty-eight (22.2\%) were lost to follow-up, $17(7.9 \%)$ were transferred out without evaluation and 14 (6.5\%) patients died. (Figure 2)

The overall TSR was 72.9\%. The highest TSR was observed in Azare prison (80.6\%) and the lowest in Misau prison (52.2\%) over the five year-period. The difference in the TSR across the five prisons formation was not statistically significant $p>0.05$ (Figure 3 )

The trend in TSR of all TB patients increased from (77.1\%) in 2014 to $(74.1 \%)$ in 2015 and decrease to (69.2\%) in 2016, further down to (58.1\%) in 2017 and (58.3\%) in 2018. (Figure 4) TSR was higher in HIV negative patients (86.0\%) compared with HIV positive patients (14.0\%) $p<0.05$; EPTB $(75.0 \%)$, compared to SNPTB $(14.7 \%)$ and SPPTB $(10.3 \%)$ p $<0.05$; new TB patients $(89.7 \%)$ compared with re-treatment $(2.2 \%)$ and Unknown $(8.1 \%) p<0.05$. TSR was higher in age group $<40$ years $(73.5 \%)$ compared to $\geq 40$ years $(26.5 \%) p<0.05$ and in those with weight $\geq 60 \mathrm{~kg}(72.1 \%)$ compared to $<60 \mathrm{~kg}(27.9 \%),(p<0.05)$.

The Odds of having successful treatment outcome was higher among the younger age groups compared to older age; 20-29 years (AOR=10.5; 95\% Cl: 3.2-35.1), 30-39 years (AOR=4.2; 95\% Cl: 1.3-13.1), and higher among TB patients with heavier weight; $50-59 \mathrm{~kg}$ (AOR=9.6; 95\% Cl: 1.4-65.6), $\geq 60 \mathrm{~kg}$ (AOR= 18.6; $95 \% \mathrm{Cl}: 2.5-14.1)$, those imprisoned for $\leq 2 y e a r s$ had a 2.3 times odds of successful treatment than those who were imprisoned for $>2$ years $(A O R=2.3 ; 95 \% \mathrm{Cl}=1.1-4.9$ ). TB patients who were not co-infected with HIV were more likely to have a successful treatment outcome compared to those co-infected with HIV TB patients who were not co-infected with HIV (AOR=3.3; 95\% Cl: 1.4-7.8). TB treatment history, year of enrollment into treatment, prisons formation and TB class were not predictors of successful treatment outcomes. (Table 3)

\section{Discussion}


This paper describes TB treatment outcome and its predictors among male prison inmates in Bauchi State. We found a high treatment success rate among the inmates. This rate though high was still below the national and international recommended minimum level for treatment success. This may not be unrelated to overcrowding, the poor sanitary condition of the prisons and the mobile nature of the inmates as they are moved from one prison to another. The risk of reinfection could be high, likewise the risk of treatment interruption resulting in treatment failure.

An effective TB control programme is determine mainly by access to TB treatment. Tuberculosis treatment is free for prison inmates in Nigeria as it is for the general population but the prisons is known to cause TB treatment interruption resulting to poor treatment outcomes. The high rate of lost to followup and transferred out without evaluation negatively impacted on the TSR.

The cure rate in this study is higher than the studies conducted in Northern Ethiopian prisons and in North Shoa, Ethiopia but lower than that recorded in El-Salvador prison. ${ }^{22,30}$ The low cure rate in this study compared to the El-Salvador study could be attributed to the lower sample size in this study and the high rates of loss to follow-up and transferred-out, since there was no system to track their progress, the final treatment outcomes of these patients were not known, so were not evaluated.

All the TB patients studied were males, this is similar to the study among prison inmates in North Shoa Ethiopia. ${ }^{22}$ The prison population are predominantly male and most prisons are male only institutions, including prisons staff. Worldwide, females makes-up just $7 \%$ of prison population, and is much lower in African countries including Nigeria. ${ }^{23}$ Female inmates constitute $2 \%$ of the total prison population in Nigeria. ${ }^{23}$ Over three-fifth of the patients studied were in the age group $<40$ years, this is similar to other findings among prison inmates with TB in North Shoa, Ethiopia but lower than that found among prison inmates in North West province, South Africa and in the general population in Western Ethiopia. ${ }^{22,24,25}$ This could be so because of high mobility, high criminal activities and imprisonment among this age group.

Tuberculosis TSR provides a useful indicator of the quality of health services. A low rate suggests that infectious patients may not be receiving adequate treatment and stand the risk of developing drug resistant TB and could serve as a potential reservoir for the transmission of MDR-TB. The overall TSR for this study is lower than the WHO set target and the Nigerian national average but similar to the rate among prison inmates in Ethiopia. 22,31,32 The lower TSR in this study results from the large number of TB patients lost to follow-up and transferred-out without evaluation of their treatment outcomes, and the significant cases of TB/HIV coinfection which is a predictor for poor TB treatment outcome in this study.

We found out that age, duration of imprisonment, weight and HIV status were predictors of successful TB treatment outcome in this study. The odds of successful treatment outcome decrease with advancing age, this is comparable with the study in Zimbabwe on age-stratified tuberculosis treatment outcomes, where the elderly had a poorer treatment outcome compared to the younger patients. ${ }^{33}$ This might be due to better immune response among the younger age group and probably other associated comorbidities 
among the older age group. Duration of incarceration was significantly associated with successful treatment outcome, this is similar to the finding among inmates in Ethiopia prisons. ${ }^{22}$ This similarity could be attributed to poor adherence to treatment protocol due to difficulty of prison life and mental stress associated with prolonged incarceration. The odds of successful treatment outcome increase with heavier body weight. Similarly, a study in Ethiopia revealed that pretreatment weight category of 55.0$70.9 \mathrm{~kg}$ and $\geq 71.0 \mathrm{~kg}$ were significantly associated with successful treatment outcome. ${ }^{34}$ This might be explained by undernutrition which increases the risk of advanced TB disease and lowers immune response, resulting to poor treatment outcome. This study revealed that TB/HIV coinfection was associated with poor treatment outcome. HIV negative TB patients had a higher odds of successful treatment outcome compared to HIV positive TB patients. This is in contrast to finding among prison inmates in northern Ethiopia where HIV coinfection was not associated with treatment outcomes but similar to that in Ethiopian university hospital. ${ }^{26,34}$ This could be attributed to the immune compromised associated with both disease, poor adherence to drugs due to large daily pills intake and the negative drug-to-drug interaction between Anti-TB and Anti-Retroviral drugs.

\section{Conclusions}

This study demonstrated that TB treatment success rate among prison inmates in Bauchi state was lower than the recommended WHO target. The TSR in this study was significantly influenced by age, duration of imprisonment, pretreatment body weight and HIV status, hence to improve TB TSR among prison inmates, these factors should be the major consideration during drugs adherence, psychological and nutritional counselling and the prison authority should develop a follow-up strategy to track inmates with TB transferred-out or discharged from prison while on treatment, this has impacted negatively on the treatment outcome and has implication for TB control programme with the risk of those who interrupted treatment developing drug-resistant TB and could serve as reservoir for the transmission of resistant strains to contacts. To the best of our knowledge, this study is the first among the studied population but it is limited by our inability to add other variables of interest which were not in the TB treatment register and may influence the treatment outcome such as educational status, cigarette smoking, comorbidity, substance use as the study relied on historical records and the study used $5.4 \%$ of the national inmates' population which might affect the generalizability of the study. Dissemination meeting was held with the TB desk officers and the superintendents of the five-prisons where findings were communicated for sensitization and resolutions reached for improved treatment outcome.

\section{Abbreviations}

EPTB Extra Pulmonary Tuberculosis

HCW Healthcare Worker

HIV Human Immunodeficiency Virus 


\section{MDR Multi-Drug Resistant}

SNPTB Smear Negative Pulmonary Tuberculosis

SPPTB Smear Positive Pulmonary Tuberculosis

TB Tuberculosis

TSR Treatment Success Rate

\section{Declarations}

Ethics approval and consent to participate

Ethical approval was obtained from ethical committee of Bauchi State Ministry of Health. Consent to participate is not applicable.

\section{Availability of data and material}

The data generated and used for this research is available from the corresponding author on reasonable request

\section{Competing interest}

The authors have no conflict of interest to declare

\section{Funding}

The authors did not receive any funding for this work.

\section{Author's Contribution}

Conceptualization: Peter Amede, Faruk S.Umar

Data curation: Peter Amede, Faruk S. Umar

Formal analysis: Peter Amede, Aishat Usman, Elizabeth Adedire, Celestine Ameh, Muhammad Shakir Balogun, Chukwuma Umeokonkwo

Methodology: Peter Amede, Aishat Usman, Elizabeth Adedire, Celestine Ameh, Muhammad Shakir Balogun, Chukwuma Umeokonkwo

Writing - review and editing: Peter Amede, Aishat Usman, Elizabeth Adedire, Celestine Ameh, Muhammad Shakir Balogun, Chukwuma David Umeokonkwo

Consent for publication 
Not applicable

\section{Acknowledgement}

The authors thank the Controller and staff of the Bauchi State prisons service for their support and the research assistants for helping to abstract information from the TB registers. We also thank the Nigeria Field Epidemiology and Laboratory Training Program.

\section{References}

1. World Health Organization (WHO): World tuberculosis day; 2018

available @https//www.who.int>2018>event

Accessed 17/02/2018

2. Park K. Epidemiology of communicable diseases: Tuberculosis. Park's Textbook of Preventive and Social Medicine, $23^{\text {rd }}$ Edition; 2015. Pg176

3. World Health Organization (WHO): Global TB report. Geneva, Switzerland; 2018.

4. Reid SE, Topp SM, Turnbull ER, et al. Tuberculosis and HIV Control in sub-Saharan African prisons: 'thinking outside the cell'. J infectious Diseases. 2012; 205(suppl 2): S265-273

5. World Health Organization (WHO): Tuberculosis in prisons. Geneva; 2011

6. Baussano I, Williams BS, Nunn P, et al. Tuberculosis incidence in the prison: a systematic review. Plos Med. 2010; 7: e1000381

7. Dara M, Grzemska M, Kimerling ME, Reyes H, Zagorskiy A. Washington DC, USA. The Global Health Bureau office of Health, Infectious Disease and Nutrition (HIDN), US Agency for International Development; 2009.

8. Nolan CM, Blumberg HM, Taylor Z, et al. Controlling tuberculosis in the United States. AM J Respiratory Critical Care Medicine. 2005; 172:1169-1227.

9. World Prison Brief: The world prison population list. Institute for Criminal Policy Research (ICPR); $12^{\text {th }}$ edition. Birkbeck publishers, University of London. September, 2018. [Online database]. Available at https://www.prisonstudies.org

Accessed on $2 / 03 / 2019$

10. United Nations Office on Drugs and Crime (UNODC). Persisting challenges and emerging strengths: findings and recommendations. Vienna, Austria; 2009. Report on the UNODC prisons assessment mission to Uganda. 
11. O' Grady J, Mwaba P, Bates M, Kapata N, Zumla A. Tuberculosis in prisons in sub-Saharan Africa, a potential time bomb. S Afri Med Journal. 2011; 101(2): 107

12. O’ Grady J, Hoelscher M, Atun R, et al. Tuberculosis. S Afri Med Journal. 2011; 91(3):173-178

13. Todrys KW, Amon JJ, Malembeka G, Clayton M. Imprisoned and Imperiled: Access to HIV and TB prevention and management and denial of human rights in Zambian prisons, J Intl AIDS Society. 2011; $14: 1-11$

14. Todrys KW, Amon JJ. Criminal Justice Reform as HIV and TB prevention in African prisons. Plos Med. 2012;9:e1001215

15. WHO|Fact sheets on tuberculosis. 2018

Available @https//www.who.int>tb>publications.

Accessed 19/02/2019

16. Sia IG, Wieland ML. Current concepts in the management of tuberculosis. Elsevier Health Sciences. 2011; 84(4):348-361

17. WHO. Global tuberculosis report. Geneva Switzerland; 2015

18. Jordan TS, Davis PD. Clinical tuberculosis and treatment outcomes. International J of tuberculosis and lung disease. 2010;14(6):683-688

19. Vesosky B, Turner J. The influence of age on immunity to infection with Mycobacterium tuberculosis. Immunological reviews. 2005; 205:229-243

20. World Health Organization (WHO): Global tuberculosis control: WHO report 2011. Geneva, Switzerland.

21. World Health Organization (WHO): Global TB report: TB treatment success rate- country ranking. Geneva Switzerland; 2017.

22. Federal Ministry of Health, Nigeria. Manual of the National Tuberculosis and Leprosy Control Programme; 2010

23. Yonas AB, Teklehaimanot MN, Gebremedhin BG. Prevalence of Tuberculosis and Treatment Outcomes of Patients with Tuberculosis among inmate in Debrebirhan prison, North Shoa Ethiopia. Ethiopian $\mathrm{J}$ of Health Science. 2018; 28(3):347-354

24. Olusegun LI, Melvin OA. Prison reform and HIV and AIDS in selected Nigeria Prisons. J of International Society Research. 2009; 1(4): 20 
25. Tirhani I. Mnisi, John T, Indiran G. Factors associated with pulmonary tuberculosis outcomes among inmates in Potchefstroom prison in North West province. Southern African Journal of epidemiology and infection. 2013;28(2):96-101

26. Muluye AB, Kebamo S, Teklia T, Alemkere G. Poor treatment outcomes and its determinants among tuberculosis patients in selected health facilities in East Wollega, Western Ethiopia. PLos ONE 13(10); e0206227. Available @https://doi.org/10.1371/journal pone.0206227

27. Kelemework Adane, Spigt MG, Geert-Jan. Tuberculosis treatment outcome and predictors in northern Ethiopian prisons: A five-year retrospective analysis. BMC Pulmonary Medicine. 2018; 18(1):13

Last accessed 1/2/2019

28. Schwitters A, Kaggwa M, Omiel P, Nagadya G, Kisa N, Dalal S. Tuberculosis and treatment completion among Ugandan prison inmates. Int J Tuberc Lung DIS.2014; 18(7):781-786

29. Adegboyega O, Eyitayo A, Leeshak E, Olufemi A. Tuberculosis and the determinants of treatment outcomes in Zaria, North Western Nigeria- A- nine- year (2007-2015) epidemiological review. Journal of Medicine in the tropics. 2017; 19(2):116-122

30. Jugens R, Nowak M, Day M. HIV and Incarceration: Prisons and detention. J Int AIDS Soc.2011; 14:26 doi: 10.1186/1758-2652-14-26 [PMC free article] [PubMed][crossRef][Google Scholar]

31. Ayala G, Garay J, Aragon M, Decroo T, Zachariah R. Trends in tuberculosis notification and treatment outcomes in prisons: a country wide assessment in El Salvador from 2009-2014. Rev Panam Salud publica. 2016; 39(1):38-43

32. World Health Organization (WHO): TBFACTS.ORG; End TB Strategy 2016-2035. Available @https://www.tbfacts.org>end-tb

Last accessed 11/01/2019

33. World Health Organization (WHO). Global tuberculosis report. Geneva; 2017 Available @http://www.who.int/tb/publications/global_report/en/

Last accessed 12/12/2018

34. Ncube RT, Takarinda KC, Zishiri $C$ et al. Age-stratified tuberculosis treatment outcomes in Zimbabwe: are we paying attention to the most vulnerable? Public Health Action. 2017; 7(3): 212-217

35. Minaleshewa B, Yiman B, Hailay A, Biruk S, Zewdu F. Treatment outcomes of Tuberculosis and associated factors in an Ethiopian University Hospital. Advances in Public Health. 2016; Vol. 2016, Article ID 8504629, 9 pages. Available at http://dx.doi.org/10.1155/2016/8504629 


\section{Tables}

Table 1: Sociodemographic characteristics of TB patients treated in five main prisons in Bauchi State from January 2014 to December 2018 (n=216)

\begin{tabular}{|c|c|c|}
\hline Variable & & Frequency(\%) \\
\hline \multicolumn{3}{|c|}{ Age (years) } \\
\hline & $20-29$ & $64(29.6)$ \\
\hline & $30-39$ & $68(31.5)$ \\
\hline & $40-49$ & $50(23.2)$ \\
\hline & $\geq 50$ & $34(15.7)$ \\
\hline \multicolumn{3}{|l|}{ Weight (kg) } \\
\hline & $30-39$ & $8(3.7)$ \\
\hline & $40-49$ & $55(25.5)$ \\
\hline & $50-59$ & $89(41.2)$ \\
\hline & $\geq 60$ & 64 (29.6) \\
\hline \multicolumn{3}{|l|}{ Prison formation } \\
\hline & Azare & 40 (18.5) \\
\hline & Bauchi & $97(44.9)$ \\
\hline & Jama'are & 30 (13.9) \\
\hline & Misau & $24(11.1)$ \\
\hline & Ningi & 25 (11.6) \\
\hline \multicolumn{3}{|c|}{ Year of enrolment } \\
\hline & 2014 & $54(25.0)$ \\
\hline & 2015 & 64 (29.6) \\
\hline & 2016 & $42(19.5)$ \\
\hline & 2017 & 34 (15.7) \\
\hline & 2018 & $32(10.2)$ \\
\hline \multicolumn{3}{|c|}{ Duration of imprisonment } \\
\hline & $\begin{array}{l}\leq 2 \text { years } \\
>2 \text { years }\end{array}$ & $\begin{array}{l}134(62.0) \\
82(38.0)\end{array}$ \\
\hline
\end{tabular}

Table 2: Clinical characteristics of TB patients treated in five main prison formation in Bauchi State from January 2014 to December $2018(\mathrm{n}=216)$ 


\begin{tabular}{cc}
\hline Variable & Frequency (\%) \\
\hline Treatment history & $188(87.0)$ \\
New & $6(2.8)$ \\
Retreatment & $2(10.2)$ \\
Unknown &
\end{tabular}

HIV status

Positive

$46(21.3)$

Negative

$170(78.7)$

TB class

$\begin{array}{lr}\text { SPPTB } & 152(70.3) \\ \text { SNPTB } & 43(19.9) \\ \text { EPTB } & 21(9.8)\end{array}$

Table 3: Predictors of successful treatment outcome of tuberculosis in five main prisons in Bauchi State from January 2014 to December 2018

\begin{tabular}{|c|c|c|c|c|}
\hline ariables & Successful n (\%) & Unsuccessful n (\%) & $\mathrm{cOR}$ & $\mathrm{aOR}$ \\
\hline ye (years) & & & & \\
\hline $20-29$ & $51(79.7)$ & $13(20.3)$ & $8.2(3.2-21.0)^{*}$ & $10.5(3.2-35.1)^{*}$ \\
\hline $30-39$ & $49(72.1)$ & 19 (27.9) & $5.4(2.2-13.2)^{*}$ & $4.2(1.3-13.1)^{*}$ \\
\hline $40-49$ & $25(50.0)$ & $25(50.0)$ & $2.1(0.8-5.2)$ & $2.8(0.1-0.7)^{*}$ \\
\hline 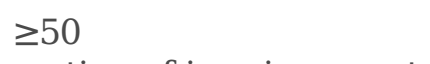 & $11(32.3)$ & $23(67.7)$ & 1 & 1 \\
\hline $\begin{array}{l}\text { uration of imprisonment } \\
\leq 2 \text { years }\end{array}$ & 99 (72.8) & 35 (43.8) & $3.4(1.9-6.2)^{*}$ & $2.6(1.3-5.4)^{*}$ \\
\hline$>2$ & $37(27.2)$ & $45(56.2)$ & 1 & 1 \\
\hline IV status & & & & \\
\hline Positive & $19(14.0)$ & $27(33.7)$ & 1 & 1 \\
\hline $\begin{array}{l}\text { Negative } \\
\text { eight (kg) }\end{array}$ & $117(86.0)$ & $47(66.3)$ & $4.0(1.3-12.1)^{*}$ & $3.3(1.4-7.8){ }^{*}$ \\
\hline $30-39$ & $2(25.0)$ & $6(75.0)$ & 1 & 1 \\
\hline $40-49$ & $10(18.2)$ & 45 (81.8) & $1.5(0.3-8.6)$ & $0.7(0.1-4.9)$ \\
\hline $50-59$ & 71 (79.8) & $18(20.2)$ & $0.1(0.1-0.5)^{*}$ & $9.6(1.4-65.6)^{*}$ \\
\hline $\begin{array}{l}\geq 60 \\
\text { ison formation }\end{array}$ & 55 (85.9) & $9(14.1)$ & $0.1(0.1-0.3)^{*}$ & $18.6(2.5-14.1)$ * \\
\hline Bauchi & $63(46.2)$ & 34 (42.5\%) & $1.4(0.6-3.2)$ & $0.8(0.3-2.4)$ \\
\hline Jama'are & $16(11.8)$ & $14(17.5)$ & $2.3(0.9-6.3)$ & $0.4(0.1-1.6)$ \\
\hline Misau & $11(8.1)$ & $13(16.2)$ & $3.1(1.1-9.0)^{*}$ & $2.3(0.1-1.4)$ \\
\hline Ningi & $17(12.5)$ & $8(10.0)$ & $1.2(0.4-3.7)$ & $0.6(0.1-2.5)$ \\
\hline Azare & $29(21.4)$ & 11 (13.8) & 1 & 1 \\
\hline
\end{tabular}


* Statistically significant; cOR-Crude Odds Ratio; aOR-Adjusted Odds Ratio

\section{Figures}

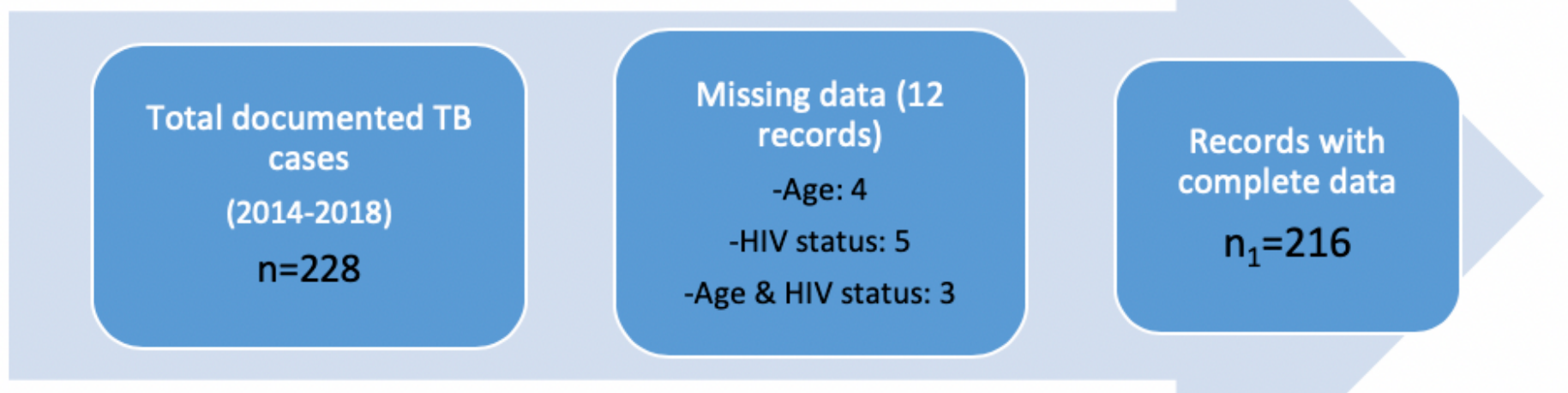

\section{Figure 1}

Data collection flowchart

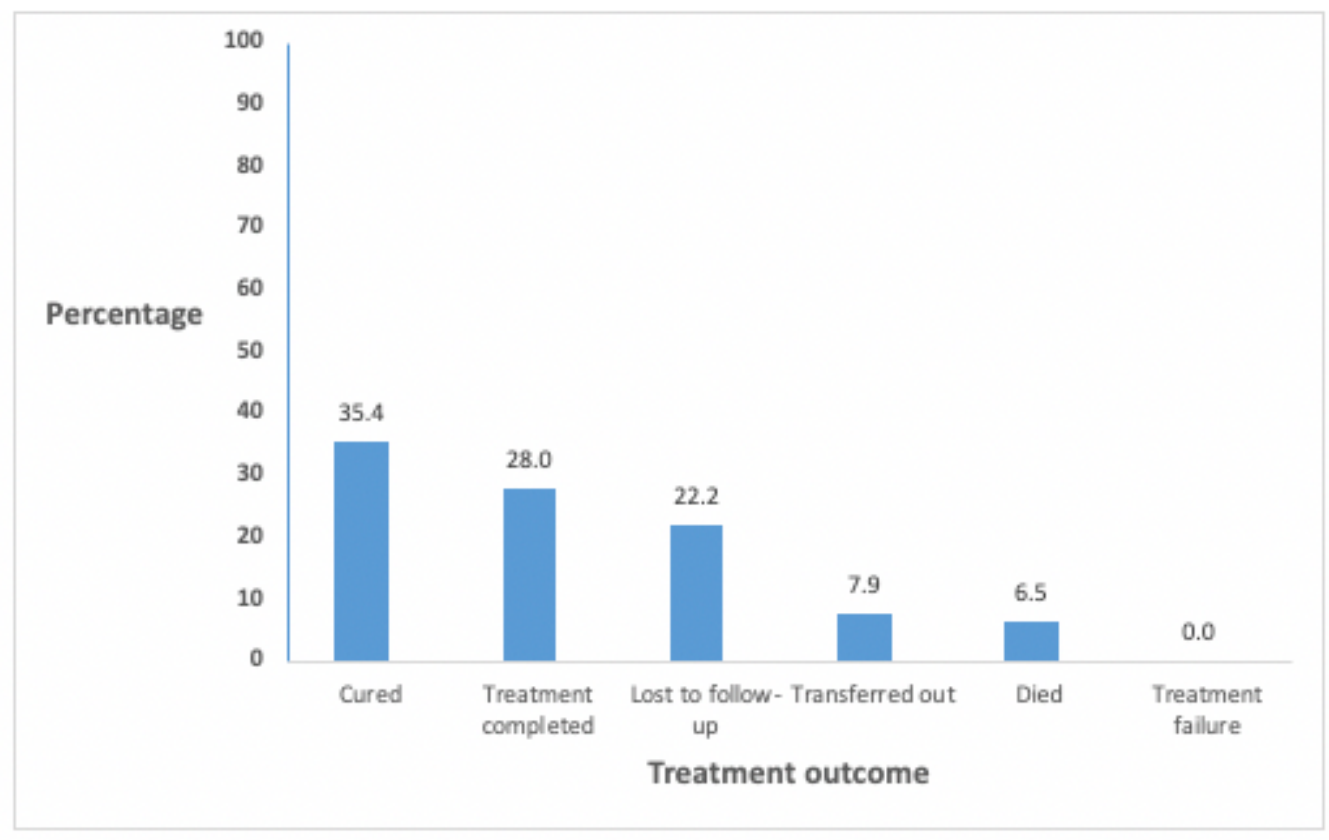

Figure 2 


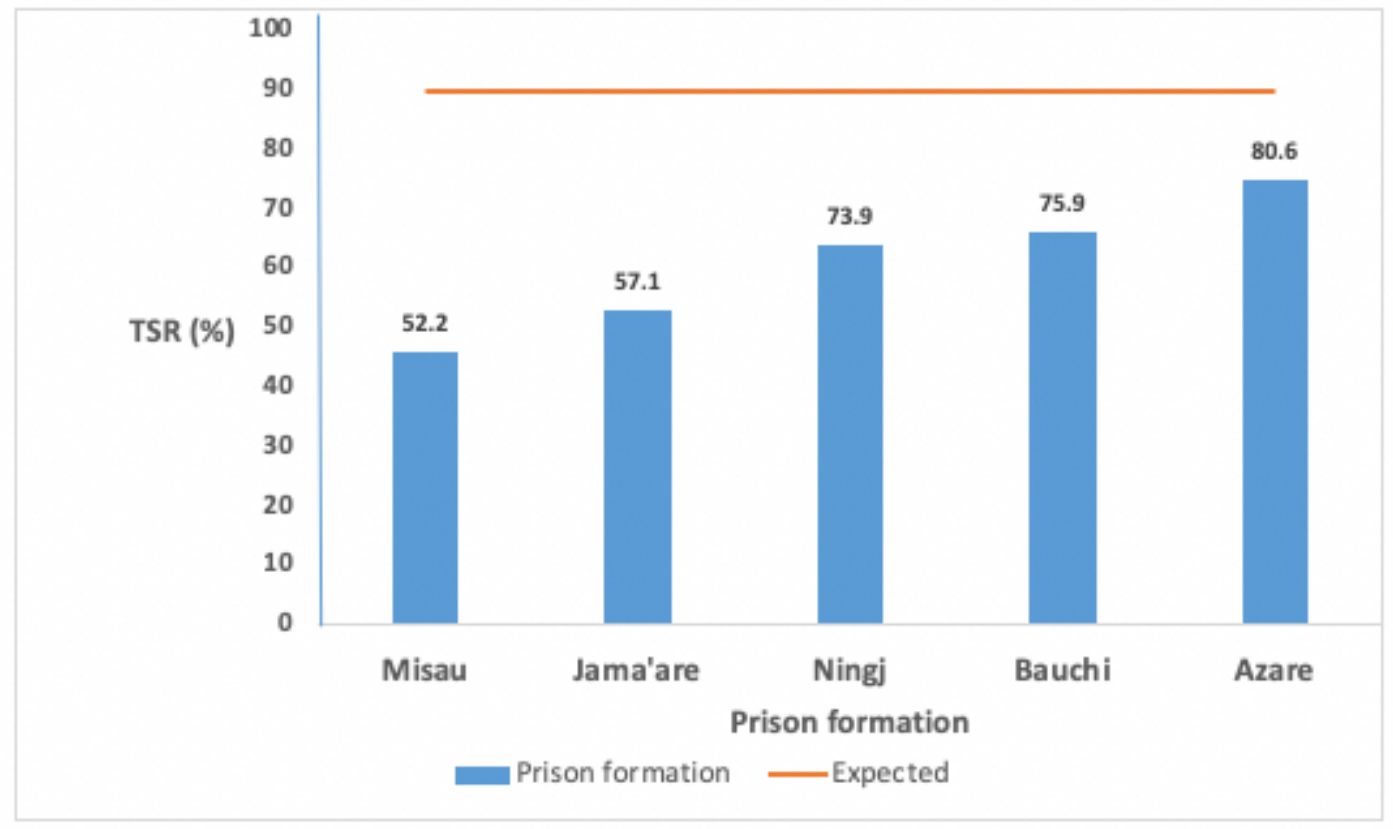

Figure 3

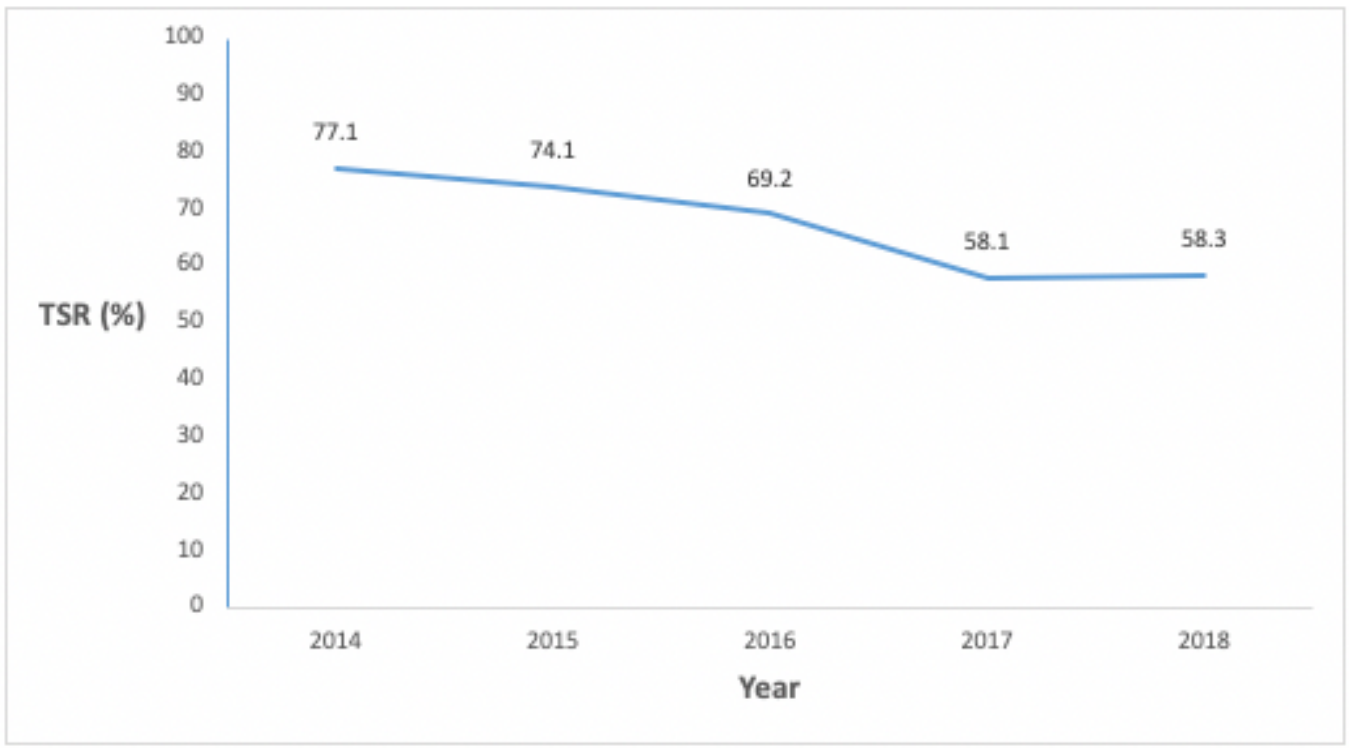

Figure 4 